\title{
Cellular Automaton Model of a Tumor Tissue Consisting of Tumor Cells, Cytotoxic T Lymphocytes (CTLs), and Cytokine Produced by CTLs
}

\author{
TOShiaki Takayanagi, ${ }^{\dagger, \text { in }}$ Hidenori Kawamura ${ }^{\dagger \dagger}$ \\ and AzUma OHUCHI ${ }^{\dagger}$
}

\begin{abstract}
We propose a cellular automaton model of a tumor tissue consisting of a square lattice, tumor cells, cytotoxic T lymphocytes (CTLs) and cytokine. Considering that CTLs circulate in vivo and migrate into a tumor tissue, the square is open and CTLs move through the square. By calculating the cellular automaton model, we obtained these results: the growth of a mass of tumor cells surrounded by CTLs; the rejection of tumor cells by CTLs; and an approximate equilibrium between tumor cells and CTLs. Analysis of the results indicates that the attachment between tumor cells and CTLs is important for the rejection of tumor cells by CTLs.
\end{abstract}

\section{Introduction}

It is known that the immune system defends humans from infections and malignant tumors ${ }^{1), 2)}$. However, it is also known that the immune system plays a causal role in many kinds of diseases, e.g., allergic diseases and autoimmune diseases ${ }^{1), 2}$. Consequently we must understand the immune system better in order to treat infections, malignant tumors and diseases caused by allergic or autoimmune response. The immune system has also attracted the attention of information engineers, because the immune system is considered to be a good natural information system. For analysis of the immune system or immune reactions, there are two methods: a model defined by equations ${ }^{3) \sim 6)}$, and a cellular automaton $(\mathrm{CA})$ model $^{7) \sim 9)}$.

The models of tumor immunity ${ }^{5), 6)}$ are considered complicated and we considered whether tumor immunity consisting of CTLs and tumor cells could be modeled. Hence, we have proposed a model of tumor immunity consisting of CTLs and tumor cells defined by equations ${ }^{3)}$, which demonstrates the tumor rejection by CTLs and the growth of a tumor mass surrounded by CTLs. However, the model ${ }^{3)}$

$\dagger$ Inazumi Park Hospital

$\dagger \dagger$ Laboratory of Harmonious Systems Engineering, Research Group of Complex Systems Engineering, Division of Synergetic Information Science, Graduate School of Information Science and Technology, Hokkaido University

is Presently with Inazumi Memorial Hospital, Caress Sapporo demonstrates neither the sneaking through phenomenon (in animal experiments, inoculation with a small number of tumor cells often leads to progressive tumor growth, inoculation with a medium number of tumor cells results in rejection, inoculation with a large number of tumor cells break through the immune defence and tumor growth) ${ }^{5), 6)}$ nor tumor dormancy (a state in which potentially lethal tumor cells persist for a long time with little or no increase in the tumor cell population) ${ }^{6)}$. To extend our study in tumor immunity, we have considered the introduction of CTL movement, cytokine including chemokine, attachment of CTLs to tumor cells into modeling of tumor immunity. For introduction of them, modeling by CA is considered better than that by equations. Therefore, we propose a CA model of tumor immunity and investigate the dynamics of the model in this paper.

The previous model ${ }^{3)}$ and the CA model proposed here are based on the mechanisms of interaction between tumor cells and tumorspecific CTLs. Namely, tumor cells proliferate automatically, they are killed by CTLs, CTLs proliferate by stimuli of tumor cells, and CTLs die automatically. Furthermore, cytokine and movement of CTLs are introduced to the CA model. Using the CA model, we investigate how CTLs contact a tumor tissue, and reject it or fail to reject it.

\section{Modeling}

\subsection{Development, Differentiation and} Circulation of T Lymphocyte

Before modeling, we briefly describe the de- 


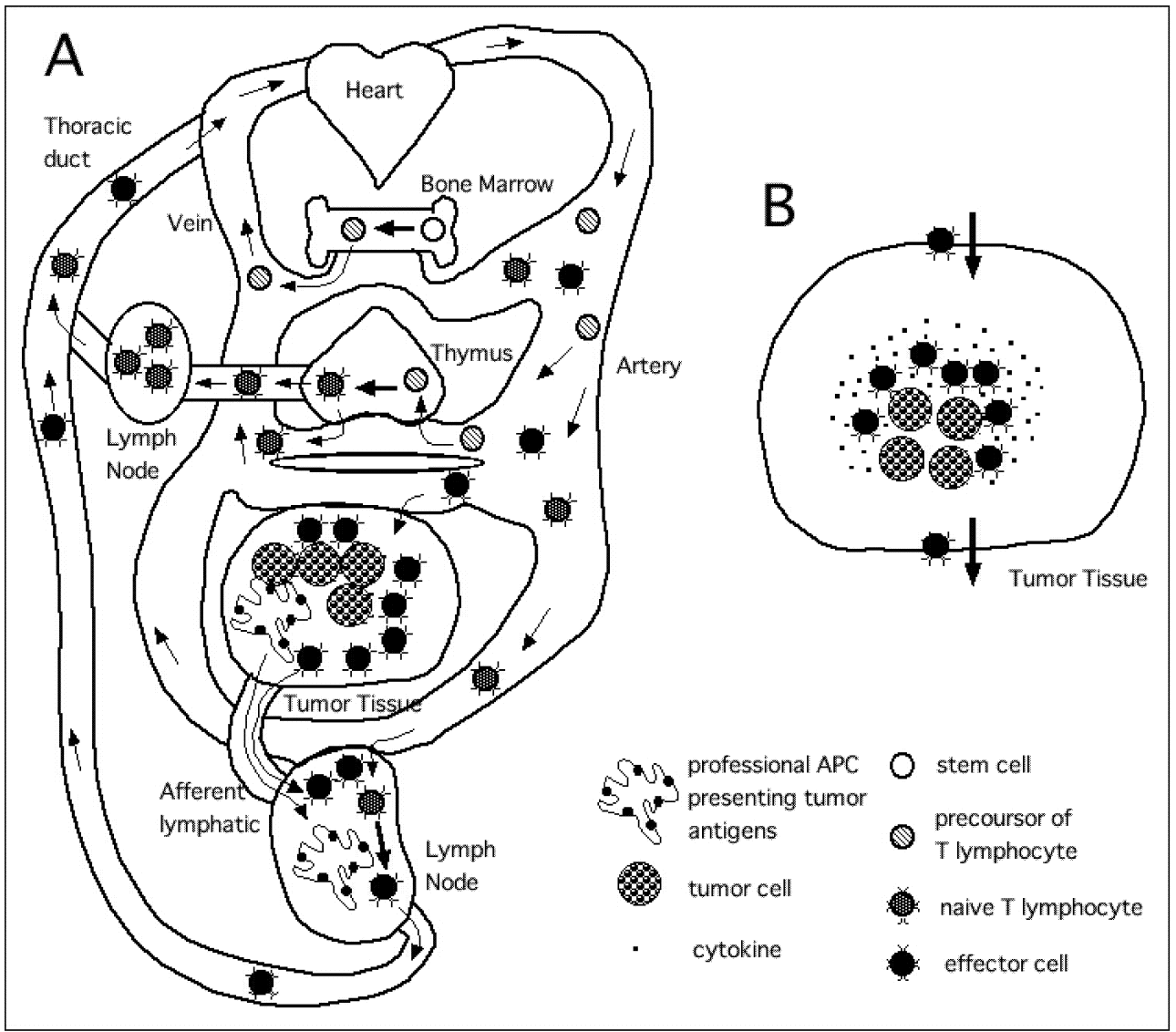

Fig. 1 In A, development, differentiation and circulation of $\mathrm{T}$ lymphocyte. In $\mathrm{B}$, tumor tissue.

velopment, differentiation and circulation of $\mathrm{T}$ lymphocytes below and in Fig. $1 \mathrm{~A}^{1), 2)}$.

Like all blood cells, T lymphocytes including CTLs arise from stem cells in the bone marrow. T lymphocyte precursors differentiated from stem cells leave the bone marrow and migrate via the bloodstream to the thymus, where they mature.

After migration of $\mathrm{T}$ lymphocyte precursors into the thymus, the cells start $\mathrm{T}$ cell receptor (TCR) gene rearrangement, i.e., a continuous V-J or V-D-J coding block encoding a variable domain in TCR is made from multiple noncontiguous V, D and J segments with nucleotide insertion and deletion at junctional sites (VJ, D-J and V-D). Consequently, T lymphocyte produces TCR with a unique antigen-binding structure during positive and negative selection in the thymus. There may be $10^{9}$ or more different $\mathrm{T}$ lymphocyte clones in an individual. As a result, naive $\mathrm{T}$ lymphocytes (in general, $\mathrm{CD} 4^{+}$ $\mathrm{T}$ lymphocytes or $\mathrm{CD}^{+} \mathrm{T}$ lymphocytes) with functional receptors exit the thymus and enter peripheral lymphoid tissues.

Naive T lymphocytes from the thymus enter a lymph node and may be activated by antigens that are transported to that node. If a naive $\mathrm{T}$ lymphocyte does not encounter an antigen specific to itself, the naive $\mathrm{T}$ lymphocyte exits through an efferent lymphatic vessel, reenters circulation, and homes to another lymph node. If the naive $\mathrm{T}$ lymphocyte does recognize an antigen specific to itself, the naive $\mathrm{T}$ lymphocyte changes into an effector $\mathrm{T}$ lymphocyte (in general, to a helper $\mathrm{T}$ lymphocyte in the case of $\mathrm{CD}^{+} \mathrm{T}$ lymphocytes; to a CTL in the case of $\mathrm{CD}^{+} \mathrm{T}$ lymphocytes) or a memory $\mathrm{T}$ lymphocyte, and the effector and memory $\mathrm{T}$ lymphocytes exit lymph nodes and preferentially home to inflamed peripheral tissues, e.g. a tumor tissue, where they are needed to eliminate antigens.

Effector and memory $\mathrm{T}$ lymphocytes preferentially home to inflammatory sites, because 
cytokines produced in response to inflammatory stimuli enhance the expression of adhesion molecules on the endothelial cells of vessels. These adhesion molecules mediate the binding of effector and memory $\mathrm{T}$ lymphocytes to the blood vessel walls at inflammatory sites, and the $\mathrm{T}$ lymphocytes subsequently migrate into the inflamed tissue.

When effector and memory $\mathrm{T}$ lymphocytes encounter their specific antigen within a peripheral tissue, they are stimulated, and their affinities for antigen-presenting cells (APCs) and for extracellular matrix proteins are increased. As a result, the antigen-stimulated effector $\mathrm{T}$ lymphocytes are preferentially retained at sites of antigen persistence in peripheral tissues and eliminate the antigens. T lymphocytes that migrate into peripheral tissues but do not see their specific antigen may enter the lymphatics and return to circulation via the thoracic duct.

The patterns of lymphocyte recirculation, i.e., the continuous movement of $\mathrm{T}$ lymphocytes via bloodstream and lymphatics from one peripheral lymphoid tissue to another and onward to peripheral inflammatory sites, are governed by the expression of adhesion molecules on lymphocytes and vascular endothelial cells.

\subsection{Outline of the Model}

According to immunological studies ${ }^{1), 2)}$, tumor tissues are surrounded by $\mathrm{T}$ lymphocytes, natural killer (NK) cells and macrophages. And tumor-bearing hosts can produce antibodies against tumor antigens. Furthermore, the effector mechanisms of both cell-mediated immunity and humoral immunity are shown to kill tumor cells in vitro; however, the ability of CTLs to provide anti-tumor immunity in vivo is most clearly seen in animal experiments. Therefore, the model we describe below is based on the basic dynamics of tumor immunity, namely the dynamic whereby tumor cells are assumed to proliferate automatically and be killed by tumor-specific CTLs, and CTLs are assumed to proliferate and produce cytokine by the stimuli of tumor cells and die naturally. Furthermore, concerning the circulation of $\mathrm{T}$ lymphocytes in vivo, CTLs are assumed to migrate into a tumor tissue, move within it and exit it; however, they are assumed not to move if activated by the cytokine, i.e., the cytokine defined in the model acts as attractans of chemotaxis with threshold $P \_t h$. In this paper, a tumor tissue is assumed to be that shown in Fig. $1 \mathrm{~B}$.

\subsection{Modeling}

The interactions between tumor cells and CTLs are modeled as follows.

- Consider a square lattice $N \times N$ (Fig. 2).

- The state-vector of each site in the square lattice has two components:

(i) each site of the square lattice can be empty, occupied by a tumor cell or occupied by a CTL;

(ii) each site has a local concentration of cytokine produced by CTLs.

- The global state of the square lattice at step $t$ is defined as $\mathbf{S}^{t}$.

- Two kinds of neighborhoods are defined as follows (Fig. 3).

(i) The contact neighborhood of a tumor cell or a CTL at a site $(i, j)$ at step $t$ is defined as:

$$
\begin{gathered}
\mathbf{C N}_{i, j}^{t}=\left\{(k, l) \in N^{2}|| k-i \mid \leq 1,\right. \\
|l-j| \leq 1 \text { and }(k, l) \neq(i, j)\} .
\end{gathered}
$$

(ii) The proliferation neighborhood of a tumor cell or a CTL at a site $(i, j)$ and time $t$ is defined as:

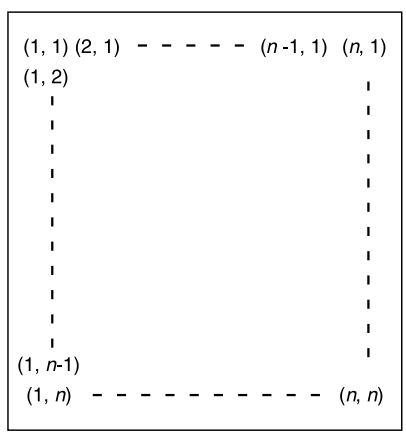

Fig. 2 Square lattice $N \times N$.
A

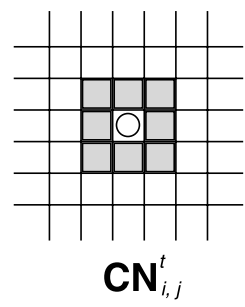

B

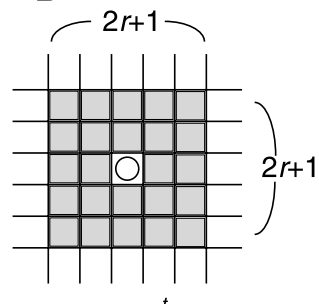

$\mathbf{P N}_{i, j}^{t}$
Fig. 3 In A, eight gray squares show $\mathbf{C N}_{i, j}^{t}$ of a cell (circle). In $\mathrm{B}$, twenty four gray squares show $\mathbf{P N}_{i, j}^{t}$ of a cell (circle) with $r=2$. 


$$
\begin{aligned}
& \mathbf{P N}_{i, j}^{t}=\left\{(k, l) \in N^{2} \| k-i \mid \leq r,\right. \\
& |l-j| \leq r,(k, l) \neq(i, j) \text { and } r \geq 1\} .
\end{aligned}
$$

In this model, a tumor cell is assumed to duplicate constantly and to be killed by one or more CTLs. A CTL is assumed to proliferate and produce cytokine by the stimuli of one or more tumor cells and is assumed to die at a constant rate. Furthermore, a CTL is assumed to migrate into a tumor tissue from the bloodstream, move within the tumor tissue, and exit the tumor tissue ${ }^{1), 2)}$. Therefore, we assign Rules 1 to 8 .

- Rule 1: A tumor cell duplicates with probability $p_{1}$. One of its daughter cells occupies the original site $(i, j)$, and the other daughter cell randomly occupies one of the empty sites in the original cell's $\mathbf{P} \mathbf{N}_{i, j}^{t}$. If there is no empty site in its $\mathbf{P} \mathbf{N}_{i, j}^{t}$, it does not duplicate.

- Rule 2: A tumor cell at a site $(i, j)$ surrounded by a CTL in its $\mathbf{C N}_{i, j}^{t}$ dies with probability $p_{2}$; that is, a tumor cell at a site $(i, j)$ surrounded by $m$ CTLs in its $\mathbf{C N}_{i, j}^{t}$ dies with probability $1-\left(1-p_{2}\right)^{m}$. If a tumor cell at a site $(i, j)$ dies, one of the surrounding CTLs in its $\mathbf{C N}_{i, j}^{t}$ moves to the site $(i, j)$.

- Rule 3: A CTL at a site $(i, j)$ surrounded by a tumor cell in its $\mathbf{C N}_{i, j}^{t}$ duplicates with probability $p_{3}$; that is, a CTL at a site $(i, j)$ surrounded by $m$ tumor cells in its $\mathbf{C N}_{i, j}^{t}$ duplicates with probability $1-\left(1-p_{3}\right)^{m}$. One of its daughter cells occupies the original site $(i, j)$, and the other daughter cell randomly occupies one of the empty sites in the original cell's $\mathbf{P} \mathbf{N}_{i, j}^{t}$. However, if there is no empty site in its $\mathbf{P} \mathbf{N}_{i, j}^{t}$, it does not duplicate.

- Rule 4: A CTL dies with probability $p_{4}$. If a CTL at a site $(i, j)$ dies, the site $(i, j)$ becomes empty.

- Rule 5: A CTL at a site $(i, j)$ surrounded by a tumor cell in its $\mathbf{C N}_{i, j}^{t}$ produces cytokine with probability $p_{5}$; that is, a CTL at a site $(i, j)$ surrounded by $m$ tumor cells in its $\mathbf{C N}_{i, j}^{t}$ produces cytokine with probability $1-\left(1-p_{5}\right)^{m}$. The amount of the cytokine produced by a CTL is constant.

- Rule 6: The concentration of cytokine decreases naturally, and the cytokine at a site $(i, j)$ diffuses to the surrounding sites, i.e., $\mathbf{C N}_{i, j}^{t}$. Therefore, when is the concentra- tion of the cytokine at a site $(i, j)$ at step $t$, then we obtain the equation below.

$$
\begin{aligned}
{[P]_{i, j}^{t+1} } & =[P]_{i, j}^{t} \\
& +p_{6} \cdot \sum_{k=i-1}^{i+1} \sum_{l=j-1}^{j+1}\left([P]_{k, l}^{t}-[P]_{i, j}^{t}\right) \\
& -p_{7} \cdot[P]_{i, j}^{t}+\frac{Q}{v}
\end{aligned}
$$

where $p_{6}$ and $p_{7}$ are constant, $v$ is the volume of a site $(i, j)$, and $Q$ is the amount of cytokine produced by a CTL. If cytokine is not produced at a site $(i, j)$ at step $t$, we obtain the equation below.

$$
\begin{aligned}
{[P]_{i, j}^{t+1} } & =[P]_{i, j}^{t} \\
& +p_{6} \cdot \sum_{k=i-1}^{i+1} \sum_{l=j-1}^{j+1}\left([P]_{k, l}^{t}-[P]_{i, j}^{t}\right) \\
& -p_{7} \cdot[P]_{i, j}^{t}
\end{aligned}
$$

- Rule 7: A CTL appears in an empty site between $(1,1)$ and $(n, 1)$ randomly with probability $p_{8}$. If there is no empty site in the line, a CTL does not appear.

- Rule 8: A CTL at a site $(i, j)$ moves to a site $(i, j+1)$ with probability $p_{9}$. If a site $(i, j+$ 1) is occupied, or if $[P]_{i, j}^{t}>P_{-} t h$, then the CTL at a site $(i, j)$ does not move. A CTL at a site $(i, n)$ disappears with probability $p_{9}$.

- The algorithm of the system is as follows:

(1) Set $\mathbf{S}^{0}$.

(2) Update $\mathbf{S}^{t}$ by updating all states in the system with Rules 1 to 8 .

(3) $t=t+1$.

(4) IF $t=t_{M A X}$, then stop. Else (2).

\section{Results}

\subsection{Natural Course of Tumor Growth}

A tumor tissue in vivo is surrounded by $\mathrm{T}$ lymphocytes, NK cells and other kinds of cells of the immune system; however, the tumor tissue continues growing in size. Figure 4 shows the results of simulation, expressing the natural course of tumor growth, i.e., the growth of a mass of tumor cells surrounded by CTLs.

\subsection{Rejection of Tumor Cells by CTLs}

Recently, anti-tumor therapy using the immune system has been the subject of much researched. The representative therapy is adaptive transfer of tumor-specific CTLs obtained from a patient with a tumor back to the same patient after activation and proliferation of the 


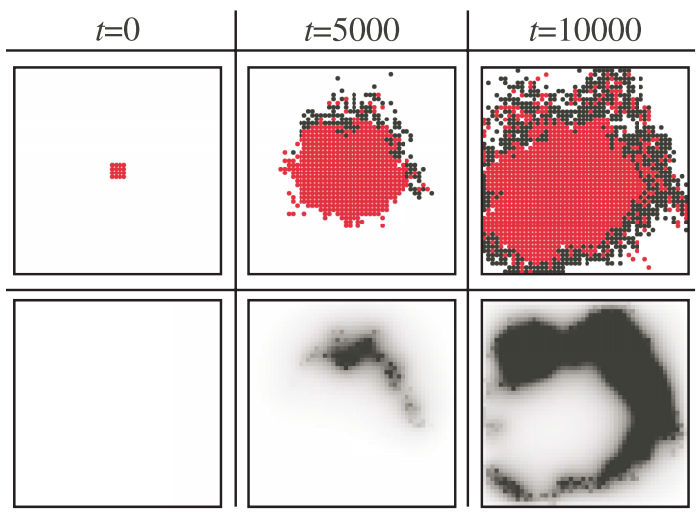

Fig. 4 Simulation of natural course of tumor growth. In the upper row, tumor cells (red circles) proliferate surrounded by CTLs (black circles). In the lower row, the concentration of cytokine is expressed by the degree of black. The concentration of cytokine is high where CTLs interact with tumor cells. The results are obtained using parameters with these values: $N=50, r=2$, $p_{1}=0.001, p_{2}=0.0002, p_{3}=0.015, p_{4}=0.02$, $p_{5}=0.2, p_{6}=0.05, p_{7}=0.01, p_{8}=0.05$, $p_{9}=0.2, Q=1, v=1, P_{\text {_th }}=1$.

CTLs with cytokines in vitro.

For the simulation of tumor rejection, the initial number of tumor cells is larger than in Fig. 4. After simulations with various values of the killing rate of a tumor cell by a CTL $\left(p_{2}\right)$, the proliferation rate of a CTL $\left(p_{3}\right)$ and the natural death rate of a CTL $\left(p_{4}\right)$, we obtained the results of simulation expressing the rejection of tumor cells by CTLs in Fig. $\mathbf{5}$.

\subsection{Approximate Equilibrium between Tumor Cells and CTLs}

We have obtained some results expressing an approximate equilibrium between a few tumor cells and a few CTLs after the decrease of tumor cells by CTLs (Fig. 6). Because CTLs move in the square lattice, CTLs are able to kill tumor cells, even if the number of tumor cells is small. However, CTLs are not able to reject tumor cells in Fig. 6.

\subsection{The Effect of Cytokine}

Next, for investigation of the effect of cytokine on the rejection of tumor cells by CTLs, we calculated the model using parameters with the same values except $Q$ as in Fig. 5. In Fig. $5, Q=1$; in Fig. 7, $Q=0$. In Fig. 5, CTLs surround a small number of tumor cells at $t=6,000$; however, in Fig. 7, CTLs do not always because the CTLs move. As a result, small populations of tumor cells and CTLs remain at $t=8,000$ and 10,000 in Fig. 7. The difference between in Fig. 5 and Fig. 7 indicates
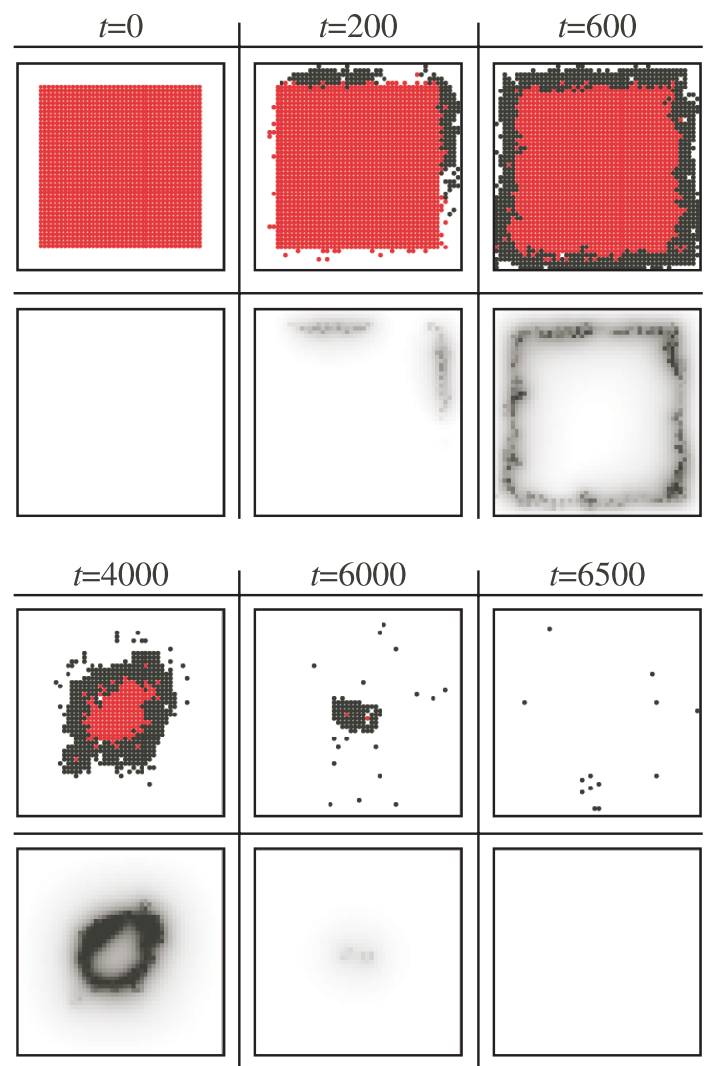

Fig. 5 Simulation of the rejection of tumor cells by CTLs. In the upper row, tumor cells (red circles) proliferate surrounded by CTLs (black circles). In the lower row, the concentration of cytokine is expressed by the degree of black. The concentration of cytokine is high where CTLs interact with tumor cells. The results are obtained using parameters with these values: $N=50, r=2, p_{1}=0.001, p_{2}=0.0006$, $p_{3}=0.045, p_{4}=0.0067, p_{5}=0.2, p_{6}=0.05$, $p_{7}=0.01, p_{8}=0.05, p_{9}=0.2, Q=1, v=1$, $P_{-} t h=1$.

that the attachment between tumor cells and CTLs by cytokine is important for tumor rejection.

\section{Discussion}

This paper proposes an original CA model of a tumor tissue. The model shows three patterns: the growth of a mass of tumor cells surrounded by CTLs in Fig. 4; the rejection of tumor cells by CTLs in Fig. 5; an approximate equilibrium between tumor cells and CTLs in Fig. 6. The model consists of a square lattice $N \times N$; however, the square is open in two directions. Furthermore, CTLs enter the square, move within it, and exit it, in the same way as the circulation of CTLs in vivo. By introducing 

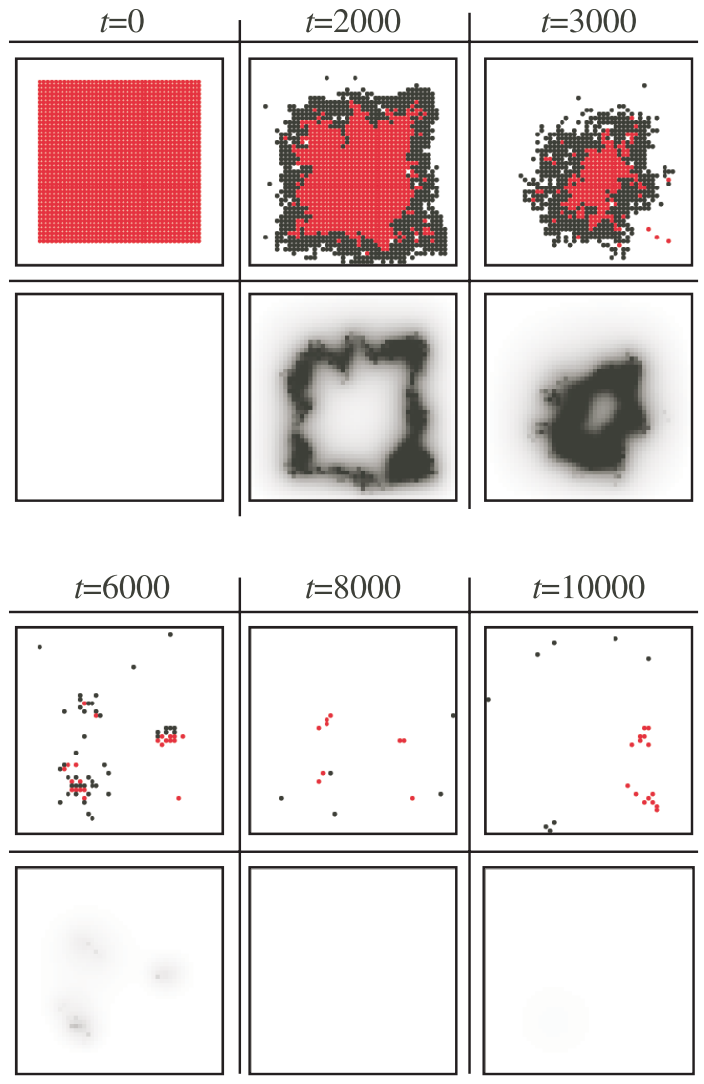

Fig. 6 Simulation of the approximate equilibrium between tumor cells and CTLs. In the upper row, tumor cells (red circles) are decreased by CTLs (black circles). In the lower row, the concentration of cytokine is expressed by the degree of black. The concentration of cytokine is high where CTLs interact with tumor cells. The results are obtained using parameters with these values: $N=50, r=2, p_{1}=0.001, p_{2}=0.001$, $p_{3}=0.02, p_{4}=0.01, p_{5}=0.2, p_{6}=0.05$, $p_{7}=0.01, p_{8}=0.05, p_{9}=0.2, Q=1, v=1$, $P \_t h=1$.

the circulation of CTLs to the model, the model can be one for a tumor tissue in vivo, although the square seems too small for the model in vivo. In the field of immunology ${ }^{1)}$, tumor tissues are surrounded by lymphocytes including T lymphocytes, and Fig. 4 demonstrates it well.

Next we discuss the attachment between tumor cells and CTLs. The attachment is assumed to be caused by cytokine produced by CTLs responding to tumor cells. Figure 5 shows the rejection of tumor cells by CTLs; Fig. 7 does not, although the only difference in parameters between Fig. 5 and Fig. 7 is that $Q=1$ in Fig. 5, whereas $Q=0$ in Fig. 7 . The difference between the results in Fig. 5 and

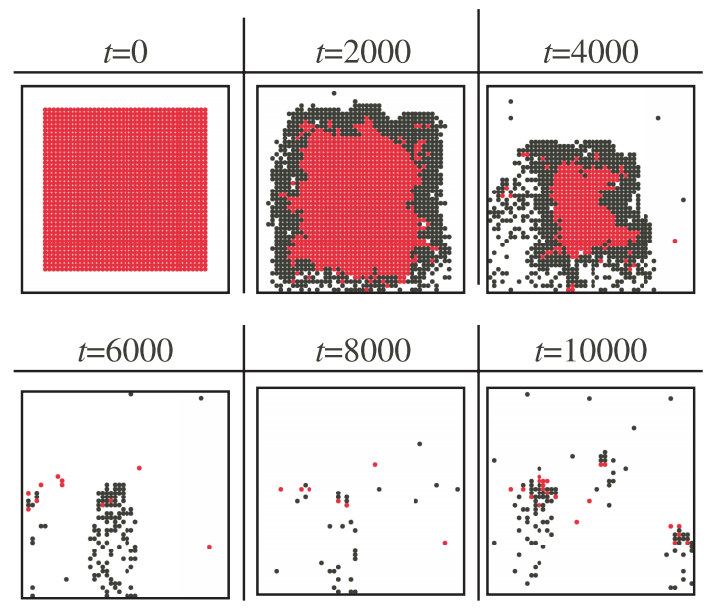

Fig. 7 Simulation of the CA model without cytokine. Tumor cells (red circles) proliferate surrounded by CTLs (black circles). The result is obtained using parameters with these values: $N=50$, $r=2, p_{1}=0.001, p_{2}=0.0006, p_{3}=0.045$, $p_{4}=0.0067, p_{5}=0.2, p_{6}=0.05, p_{7}=0.01$, $p_{8}=0.05, p_{9}=0.2, Q=0, v=1, P_{-} t h=1$.

those in Fig. 7 indicates that attachment between tumor cells and CTLs is important in the rejection of tumor cells.

Figure 6 shows an approximate equilibrium between a few tumor cells and a few CTLs, which seems tumor dormancy ${ }^{6}$ ). Before this study, we created a CA model of tumor immunity, into which CTL movement is not introduced. The CA model without CTL movement does not present an approximate equilibrium as in Fig. 6. Hence, CTL movement seems important for tumor dormancy. However, the CA model in this paper does not show sneaking through phenomenon ${ }^{5), 6)}$.

Acknowledgments We would like to express our thanks to Dr. Hiroki Shirato for his useful advise.

\section{References}

1) Abbas, A.K., Lichtman, A.H. and Pober, J.S.: Cellular and molecular immunology, 4th ed., W.B. Saunders Company (2000)

2) Goldman, L. and Bennett, J.C.: Cecil textbook of medicine, 21st ed., W.B. Saunders Company (2001)

3) Takayanagi, T. and Ohuchi, A.: A mathematical analysis of the interactions between immunogenic tumor cells and cytotoxic $\mathrm{T}$ lymphocytes, Microbiol. Immunol., Vol.45, pp.709715 (2001).

4) Takayanagi, T. and Ohuchi, A.: Computer simulations of slow progression of human im- 
munodeficiency virus infection and relapse during anti-HIV treatment with reverse transcriptase inhibitors and protease inhibitors, Microbiol. Immunol., Vol.46, pp.397-407 (2002).

5) Grossman, Z. and Berke, G.: Tumor escape from immune elimination, J. Theor. Biol., Vol.83, pp.267-296 (1980).

6) Kuznetsov, V.A., Makalkin, I.A., Taylor, M.A. and Perelson, A.S.: Nonlinear dynamics of immunogenic tumors: Parameter estimation and global bifurcation analysis, Bull. Math. Biol., Vol.56, pp.295-321 (1994).

7) Bernaschi, M. and Succi, S.: Large-scale cellular automata simulations of the immune system response, Phys. Rev. E, Vol.61, pp.1851-1854 (2000).

8) Mielke, A. and Pandey, R.B.: A computer simulation study of cell population in a fuzzy interaction model for mutating HIV, Physica A, Vol.251, pp. 430-438 (1998).

9) Bernaschi, M. and Castiglione, F.: Design and implementation of an immune system simulator, Comput. Biol. Med., Vol.31, pp.303-331 (2001).

(Received December 7, 2004)

(Revised May 25, 2005)

(Accepted June 10, 2005)

(Released March 1, 2006)

(Paper version of this article can be found in the IPSJ Transactions on Mathematical Modeling and Its Applications, Vol.47 No.SIG1(TOM14), pp.61-67.)

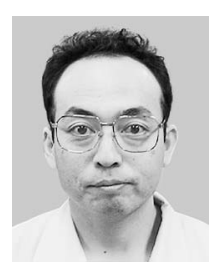

Toshiaki Takayanagi graduated from Hokkaido University Medical School in 1992. He received his Ph.D. degree in medical science and Ph.D. degree in engineering from Hokkaido University in 1998 and 2002, respectively. He works as a medical doctor of internal medicine. His research is in the field of modeling of biological reactions.

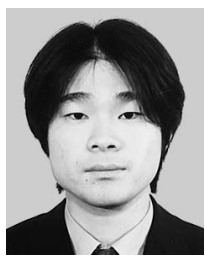

Hidenori Kawamura received his B.E. degree, M.E. degree, and D.E. degree from Hokkaido University in 1996 , 1998 and 2000 respectively. Since 2000 he has been an instructor in Graduate School of Engineering, Hokkaido University. His main research interests are complex adaptive systems, multi-agent systems, agent based simulations, artificial life and artificial intelligence.

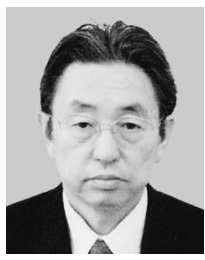

Azuma Ohuchi is a professor of Information Engineering at Hokkaido University, Sapporo, Japan. He has been developing a new field of complex systems engineering, i.e., Harmonious Systems Engineering since 1995. He has published numerous papers on systems engineering, operations research, and computer science. In addition, he is currently supervising projects on multi-agents based artificial market systems, medical informatics, autonomous flying objects, and DNA computing. Dr. Ohuchi was awarded "The 30th Anniversary Award for Excellent Papers" by the Information Processing Society of Japan. $\mathrm{He}$ is a member of Operations Research Society of Japan, Japanese Society for Artificial Intelligence, Information Processing Society of Japan, Japan Association for Medical Informatics, IEEE Computer Society, IEEE System, Man and Cybernetics Society, etc. He received Ph.D. from Hokkaido University in 1976. 